\title{
Supersymmetry breaking from complex linear superfields
}

\section{Pavel Kočí}

Masaryk University, Czech Republic

E-mail: pavelkoci@mail.muni.cz

We review the properties of a new mechanism for spontaneous supersymmetry breaking which is built from complex linear superfields. Using superspace higher derivative terms which deform the auxiliary field potential we find that, in addition to the standard supersymmetric vacuum, there is a new vacuum where supersymmetry is spontaneously broken and nonlinearly realized. From the Noether method we identify the chiral superfield $X$ which enters the Ferrara-Zumino supercurrent equation and show that it becomes nilpotent in the broken vacuum.

Proceedings of the Corfu Summer Institute 2015 "School and Workshops on Elementary Particle Physics and Gravity"

1-27 September 2015

Corfu, Greece

${ }^{*}$ Speaker. 


\section{Introduction and discussion}

If supersymmetry [1] is realized in nature it must be spontaneously broken at some high energy scale, since no superpartner of the Standard Model particles has been observed until now. It is yet not known how exactly supersymmetry is broken and how the breaking is mediated to the supersymmetric Standard Model, even though various mechanisms have been proposed [2]. The study of the different supersymmetry breaking mechanisms can help understand their common and also their distinct properties. In this article we focus on a novel mechanism for supersymmetry breaking which is built from complex linear superfields.

The complex linear multiplet was introduced in [3] and further studied in $[4,5,6,7,8,9,10$, 11]. In contrast with a chiral superfield, such a multiplet contains a bigger set of auxiliary fields needed to close the supersymmetry algebra off-shell, including fermion auxiliary fields. It is known that the chiral and complex linear superfields generically offer an equivalent on-shell description of the same physics [1]. However the models we study here belong to a class of theories where this duality is not valid any more. This is known to happen whenever auxiliary fields of the free theory become propagating due to higher derivative terms [12, 13, 14]. Indeed, as we will see, in this mechanism the goldstino is a previously auxiliary fermion of the free theory which becomes propagating in the broken vacuum.

Recent work in $4 \mathrm{D}, N=1$ supersymmetry $[15,16,17]$ has shown that superspace higher derivative terms containing complex linear superfields can be used to deform the auxiliary field potential in such a way that they break supersymmetry. The simplest models have the following form

$$
\mathscr{L}=-\int d^{4} \theta \bar{\Sigma} \Sigma+\frac{1}{8 f^{2}} \int d^{4} \theta D^{\alpha} \Sigma D_{\alpha} \Sigma \bar{D}^{\dot{\beta}} \bar{\Sigma} \bar{D}_{\dot{\beta}} \bar{\Sigma}
$$

where

$$
\bar{D}^{2} \Sigma=0
$$

This mechanism is based on the existence of various different vacua arising as solutions to the auxiliary field equations of motion. On top of the standard supersymmetry preserving solution there is a new vacuum where the scalar auxiliary field gets a nonvanishing vev. Moreover, one of the auxiliary fermions starts propagating in the new supersymmetry breaking vacuum and is the Goldstone mode of the broken global supersymmetry. One interesting feature of this type of supersymmetry breaking is that in the broken vacuum supersymmetry is directly non-linearly realized and there is no superpartner for the goldstino. This is in contrast to supersymmetry breaking from chiral superfields where one needs to impose a nilpotency condition to get a non-linear realization of supersymmetry or decouple the massive superpartner of the goldstino [18, 19, 20, 21]. Finally, since the main purpose of supersymmetry breaking is to generate mass splitting between superpartners, we will see how one can modify the model (1.1) to generate a mass term for the otherwise massless scalar of the complex linear multiplet $[15,17]$. For models with chiral superfields see $[22,23,24,25,26,27,28,29,30,31,32,33,34,35,36,37]$ and for modified complex linear superfields see [38]. 
By following the Noether method we also study the supercurrent [39, 40, 41, 42, 43] of the model (1.1). We have found that one can consistently write the supercurrent equation in the form

$$
\bar{D}^{\dot{\alpha}} J_{\alpha \dot{\alpha}}=D_{\alpha} X
$$

and identify both the Ferrara-Zumino multiplet $\left(J_{\alpha \dot{\alpha}}\right)$ and the chiral superfield $X$. This shows that one can consistently couple the Lagrangian (1.1) to old-minimal supergravity. In addition, we show that the $X$ superfield in (1.3) becomes indeed a nilpotent chiral goldstino superfield as was advocated in [21] to hold for every supersymmetry breaking theory in the low energy limit.

\section{Chiral goldstino superfield}

In this section we review supersymmetry breaking by a chiral superfield, and we see how the decoupling of the sgoldstino leads to a non-linear realization of supersymmetry. For a chiral superfield $\Phi$, which satisfies $\bar{D}_{\dot{\alpha}} \Phi=0$, the component fields are defined as

$$
\Phi\left|=z, D_{\alpha} \Phi\right|=\rho_{\alpha}, D^{2} \Phi \mid=N .
$$

The simplest model that breaks supersymmetry is

$$
\begin{aligned}
\mathscr{L} & =\int d^{4} \theta \bar{\Phi} \Phi-\left\{c \int d^{2} \theta \Phi+\text { c.c. }\right\}= \\
& =\frac{1}{2} z \partial^{\alpha \dot{\alpha}} \partial_{\alpha \dot{\alpha}} \bar{z}+N \bar{N}-c N-c \bar{N}-i \rho_{\alpha} \partial^{\alpha \dot{\beta}} \bar{\rho}_{\dot{\beta}} .
\end{aligned}
$$

The equation of motion for the auxiliary field $N$ reads

$$
\delta \bar{N}: N=c,
$$

and the Goldstone fermion can be identified as the fermion with the supersymmetry transformation

$$
\delta \rho_{\alpha}=\varepsilon_{\alpha} c+\ldots
$$

while the vacuum energy of the theory is positive: $\langle V\rangle=c^{2}$. Both the existence of a goldstino and the positive vacuum energy implies broken global supersymmetry.

Now we want to study a more general class of models in which the sgoldstino becomes massive. These models are described by the Lagrangian

$$
\mathscr{L}=\int d^{4} \theta \bar{\Phi} \Phi-r \int d^{4} \theta \bar{\Phi}^{2} \Phi^{2}-\left\{c \int d^{2} \theta \Phi+\text { c.c. }\right\},
$$

which in components becomes

$$
\begin{aligned}
\mathscr{L} & =\frac{1}{2} z \partial^{\alpha \dot{\alpha}} \partial_{\alpha \dot{\alpha}} \bar{z}+N \bar{N}-c N-c \bar{N}-i \rho_{\alpha} \partial^{\alpha \dot{\beta}} \bar{\rho}_{\dot{\beta}} \\
& -r\left\{z \bar{z}\left(-\frac{1}{2} \partial^{\alpha \dot{\alpha}} z \partial_{\alpha \dot{\alpha}} \bar{z}-\frac{i}{2}\left(\rho_{\alpha} \partial^{\alpha \dot{\beta}} \bar{\rho}_{\dot{\beta}}+\bar{\rho}_{\dot{\beta}} \partial^{\alpha \dot{\beta}} \rho_{\alpha}\right)+N \bar{N}\right)\right. \\
& \left.+\frac{z}{2}\left(N \bar{\rho}^{\dot{\alpha}} \bar{\rho}_{\dot{\alpha}}-i \rho_{\alpha} \bar{\rho}_{\dot{\beta}} \partial^{\alpha \dot{\beta}} \bar{z}\right)+\frac{\bar{z}}{2}\left(\bar{N} \rho^{\alpha} \rho_{\alpha}+i \rho_{\alpha} \bar{\rho}_{\dot{\beta}} \partial^{\alpha \dot{\beta}} z\right)+\frac{1}{4} \rho^{\alpha} \rho_{\alpha} \bar{\rho}^{\dot{\alpha}} \bar{\rho}_{\dot{\alpha}}\right\} .
\end{aligned}
$$


We will see that once we decouple the massive complex scalar $z$ from the spectrum of the theory, we get the Lagrangian (2.2) with nonlinearly realized supersymmetry [18, 19, 20, 21]. The equation of motion for $\bar{N}$ is

$$
\delta \bar{N}: N=\frac{c}{1-r z \bar{z}},
$$

which shows that supersymmetry is broken for small $z$ fluctuations around the vacuum $z_{0}=0$. Expanding around the vacuum one finds the mass eigenvalues for the sgoldstino

$$
m^{2} \propto r .
$$

Therefore, if we formally send the curvature $r$ to infinity, we decouple the massive $z$ degrees of freedom from the theory. This will make the $r$-dependent parts to dominate the equations of motion, therefore they have to be solved independently as if they were constraints. Let us see how this happens. Varying the divergent part of the Lagrangian we find that

$$
\begin{aligned}
\delta z & : \frac{1}{4} z \partial^{\alpha \dot{\alpha}} \partial_{\alpha \dot{\alpha}} \bar{z}^{2}+\bar{z}\left(-\frac{i}{2}\left(\rho_{\alpha} \partial^{\alpha \dot{\beta}} \bar{\rho}_{\dot{\beta}}+\bar{\rho}_{\dot{\beta}} \partial^{\alpha \dot{\beta}} \rho_{\alpha}\right)+N \bar{N}\right) \\
& +\frac{1}{2}\left(N \bar{\rho}^{\dot{\alpha}} \bar{\rho}_{\dot{\alpha}}-i \rho_{\alpha} \bar{\rho}_{\dot{\beta}} \partial^{\alpha \dot{\beta}} \bar{z}\right)=0 .
\end{aligned}
$$

A non-dynamical solution to the above equation, which removes $z$, is

$$
\bar{z}=-\frac{\bar{\rho}^{\dot{\alpha}} \bar{\rho}_{\dot{\alpha}}}{2 \bar{N}}
$$

which gives the superspace constraint

$$
\Phi^{2}=0
$$

To get a formulation in superspace and impose the nilpotency constraint directly on the goldstino superfield, we modify (2.2) in the following way

$$
\mathscr{L}=\int d^{4} \theta X_{N L} \bar{X}_{N L}+\left\{\int d^{2} \theta\left(-c X_{N L}+\mathscr{C} X_{N L}^{2}\right)+c . c .\right\},
$$

where $X_{N L}$ is the chiral goldstino superfield and $\mathscr{C}$ is a chiral Lagrange multiplier. Variation of the Lagrangian leads to a set of equations

$$
\begin{aligned}
\delta \mathscr{C} & : X_{N L}^{2}=0, \\
\delta \bar{X}_{N L} & : \bar{D}^{2} \bar{X}_{N L}-c+2 \mathscr{C} X_{N L}=0 .
\end{aligned}
$$

As we have seen, using the condition (2.13) we can express $X_{N L}$ only in terms of the goldstino $\rho_{\alpha}$ and the scalar field $N$.

\section{Supersymmetry breaking and complex linear superfield}

Let us start with a free theory for the complex linear superfield

$$
\mathscr{L}=-\int d^{4} \theta \bar{\Sigma} \Sigma=
$$




$$
=\frac{1}{2} A \partial^{\alpha \dot{\alpha}} \partial_{\alpha \dot{\alpha}} \bar{A}-F \bar{F}+P^{\alpha \dot{\alpha}} \bar{P}_{\alpha \dot{\alpha}}-i \psi_{\alpha} \partial^{\alpha \dot{\beta}} \bar{\psi}_{\dot{\beta}}+\chi^{\alpha} \lambda_{\alpha}+\bar{\chi}^{\dot{\alpha}} \bar{\lambda}_{\dot{\alpha}}
$$

where the component definitions are

$$
\begin{aligned}
& \Sigma\left|=A, D^{2} \Sigma\right|=F, \bar{D}_{\dot{\alpha}} D_{\alpha} \Sigma \mid=P_{\alpha \dot{\alpha}}, \\
& \bar{D}_{\dot{\alpha}} \Sigma\left|=\bar{\psi}_{\dot{\alpha}}, D_{\alpha} \Sigma\right|=\lambda_{\alpha}, \frac{1}{2} D^{\gamma} \bar{D}_{\dot{\alpha}} D_{\gamma} \Sigma \mid=\bar{\chi}_{\dot{\alpha}} .
\end{aligned}
$$

From the structure of the free theory we can see that $\chi, \lambda, P, F$ are auxiliary fields. Now we would like to explore the properties of the theory given by (1.1). To probe vacuum structure we look at the bosonic part of the theory

$$
\begin{aligned}
\mathscr{L}_{\mathrm{B}}= & \frac{1}{2} A \partial^{\alpha \dot{\alpha}} \partial_{\alpha \dot{\alpha}} \bar{A}-F \bar{F}+P^{\alpha \dot{\alpha}} \bar{P}_{\alpha \dot{\alpha}}+\frac{1}{2 f^{2}} F^{2} \bar{F}^{2}+\frac{1}{2 f^{2}} F \bar{F} P^{\alpha \dot{\alpha}} \bar{P}_{\alpha \dot{\alpha}} \\
& +\frac{1}{8 f^{2}} P^{\alpha \dot{\alpha}} P_{\alpha \dot{\alpha}} \bar{P}^{\beta \dot{\beta}} \bar{P}_{\beta \dot{\beta}} .
\end{aligned}
$$

Varying the Lagrangian with respect to $P_{\alpha \dot{\alpha}}$ we get

$$
\bar{P}_{\alpha \dot{\alpha}}+\frac{1}{2 f^{2}} F \bar{F} \bar{P}_{\alpha \dot{\alpha}}+\frac{1}{4 f^{2}} P_{\alpha \dot{\alpha}} \bar{P}^{\beta \dot{\beta}} \bar{P}_{\beta \dot{\beta}}=0
$$

which has solution

$$
P_{\alpha \dot{\alpha}}=0
$$

The equation of motion for $F$ simplifies to

$$
\bar{F}-\frac{1}{f^{2}} F \bar{F}^{2}=0,
$$

which has two solutions

$$
\begin{aligned}
& F=0, \\
& F \bar{F}=f^{2} .
\end{aligned}
$$

The first solution preserves supersymmetry, whereas the second solution gives spontaneous supersymmetry breaking. Since supersymmetry is broken we have to verify the existence of a goldstone mode and the positive vacuum energy. Indeed, up to quadratic order to the fields the Lagrangian in the supersymmetry breaking vacuum is

$$
\mathscr{L}_{\text {Quad. }}=\frac{1}{2} A \partial^{\alpha \dot{\alpha}} \partial_{\alpha \dot{\alpha}} \bar{A}-i \psi_{\alpha} \partial^{\alpha \dot{\beta}} \bar{\psi}_{\dot{\beta}}-\frac{1}{2} f^{2}-i \lambda_{\beta} \partial^{\beta \dot{\beta}} \bar{\lambda}_{\dot{\beta}}
$$

As before, using the supersymmetry transformation we can identify the Goldstone fermion

$$
\delta \lambda_{\alpha}=\varepsilon_{\alpha} f+\ldots
$$

and verify the positive vacuum energy: $\langle V\rangle=\frac{1}{2} f^{2}$. 
It is possible to solve the equations of motion directly in superspace. Varying the Lagrangian (1.1) we find

$$
\Sigma+\frac{1}{4 f^{2}} \bar{D}^{\dot{\alpha}}\left(\bar{D}_{\dot{\alpha}} \bar{\Sigma} D^{\alpha} \Sigma D_{\alpha} \Sigma\right)=\bar{\Phi}
$$

where $\bar{\Phi}$ is the free anti-chiral superfield $\left(\bar{D}^{2} \bar{\Phi}=0\right)$. Apart from the standard supersymmetric solution $\Sigma=\bar{\Phi}$ there is also the supersymmetry breaking solution

$$
\Sigma=\bar{\Phi}+X_{N L}
$$

where $X_{N L}$ is the chiral goldstino with $c=f$.

It is a well known fact that the complex linear superfield can be generically dualized to the chiral superfield. However, this procedure can be non-trivial once there are new propagating degrees of freedom arising from higher derivative terms. Here we have seen that on top of the free chiral superfield (which is dual to the free complex linear) there is a goldstino multiplet also propagating which is verified by formula (3.11).

Now we would like to discuss one possible generalization of (1.1) that mediates mass to the scalar sector $[15,17]$. Let us study the following Lagrangian

$$
\mathscr{L}=-\int d^{4} \theta \bar{\Sigma} \Sigma+\frac{1}{8} \int d^{4} \theta U(\Sigma, \bar{\Sigma}) D^{\alpha} \Sigma D_{\alpha} \Sigma \bar{D}^{\dot{\beta}} \bar{\Sigma} \bar{D}_{\dot{\beta}} \bar{\Sigma},
$$

where $U(\Sigma, \bar{\Sigma})$ is a real positive function. The bosonic part has a form

$$
\begin{aligned}
\mathscr{L}_{\mathrm{B}}= & \frac{1}{2} A \partial^{\alpha \dot{\alpha}} \partial_{\alpha \dot{\alpha}} \bar{A}-F \bar{F}+P^{\alpha \dot{\alpha}} \bar{P}_{\alpha \dot{\alpha}}+\frac{1}{2} U(A, \bar{A}) F^{2} \bar{F}^{2}+\frac{1}{2} U(A, \bar{A}) F \bar{F} P^{\alpha \dot{\alpha}} \bar{P}_{\alpha \dot{\alpha}} \\
& +\frac{1}{8} U(A, \bar{A}) P^{\alpha \dot{\alpha}} P_{\alpha \dot{\alpha}} \bar{P}^{\beta \dot{\beta}} \bar{P}_{\beta \dot{\beta}},
\end{aligned}
$$

where $U(A, \bar{A})=U(\Sigma, \bar{\Sigma}) \mid$. As before, we find the solution $P_{\alpha \dot{\alpha}}=0$, and the equation of motion for $F$ is

$$
\bar{F}-U(A, \bar{A}) F \bar{F}^{2}=0 .
$$

For the supersymmetry breaking vacuum we have

$$
F \bar{F}=U(A, \bar{A})^{-1} .
$$

Plugging this solution into the Lagrangian, for $A$ we get

$$
\left.\mathscr{L}_{\mathrm{B}}\right|_{A}=\frac{1}{2} A \partial^{\alpha \dot{\alpha}} \partial_{\alpha \dot{\alpha}} \bar{A}-\frac{1}{2 U(A, \bar{A})} .
$$

This is a new mechanism for generating scalar potentials for $A$, and we will illustrate the properties of these potentials with two examples. For our first example we have

$$
U(A, \bar{A})=\frac{2}{f^{2}+m_{A}^{2} A \bar{A}},
$$

which gives a scalar potential

$$
V=f^{2}+m_{A}^{2} A \bar{A},
$$


and breaks supersymmetry but also gives a mass to the scalar field $A$, (which is not the sgoldstino). The second example is given by

$$
U(A, \bar{A})=\frac{2}{f^{2}+\frac{\lambda}{4 !}\left(A \bar{A}-\mu^{2}\right)^{2}},
$$

which gives rise to the scalar potential

$$
V=f^{2}+\frac{\lambda}{4 !}\left(A \bar{A}-\mu^{2}\right)^{2}
$$

In the vacuum we have $A \bar{A}=\mu^{2}$ which breaks both supersymmetry and the global $U(1)$ symmetry.

\section{Supercurrents}

In principle, the supercurrent conservation equation (which holds only on-shell) has the generic form $[40,39,41,42,43]$

$$
\bar{D}^{\dot{\alpha}} \mathscr{J}_{\alpha \dot{\alpha}}=\mathscr{Y}_{\alpha}+\mathscr{X}_{\alpha},
$$

where the supercurrent $\mathscr{J}_{\alpha \dot{\alpha}}$ is a real superfield, and the superfields $\mathscr{Y}_{\alpha}$ and $\mathscr{X}_{\alpha}$ satisfy

$$
\begin{aligned}
\bar{D}_{\dot{\alpha}} \mathscr{X}_{\alpha} & =0, & \bar{D}^{2} \mathscr{Y}_{\alpha} & =0, \\
D^{\alpha} \mathscr{X}_{\alpha}-\bar{D}^{\dot{\alpha}} \overline{\mathscr{X}}_{\dot{\alpha}} & =0, & D_{\alpha} \mathscr{Y}_{\beta}+D_{\beta} \mathscr{Y}_{\alpha} & =0 .
\end{aligned}
$$

For the Ferrara-Zumino supercurrent we have that

$$
\mathscr{Y}_{\alpha}=D_{\alpha} X, \mathscr{X}_{\alpha}=0
$$

It has been advocated in [21] that the $X$ in (4.4) flows to the nilpotent chiral superfield $X_{N L}$, in the low energy limit of supersymmetry breaking theories. In this section we find the supercurrent of the model (1.1) by using the superspace Noether procedure [40]. Then we will show that also in these models the $X$ superfield that enters the Ferrara-Zumino supercurrent equation becomes the nilpotent chiral goldstino superfield $X_{N L}$.

We start from the superspace Noether procedure. An infinitesimal superdiffeomorphism $\left(\bar{D}^{2} \delta \Sigma=\right.$ 0 ) for the complex linear superfield can be expressed in terms of unconstraint superfields $L^{\alpha}, L^{\dot{\beta} \dot{\alpha}}$ as

$$
\delta \Sigma=[i \Delta, \Sigma]=-\bar{D}^{2} L^{\alpha} D_{\alpha} \Sigma+i \bar{D}^{\dot{\alpha}} L^{\alpha} \partial_{\alpha \dot{\alpha}} \Sigma+i \bar{D}_{\dot{\beta}} L^{\dot{\beta} \dot{\alpha}} \bar{D}_{\dot{\alpha}} \Sigma .
$$

An infinitesimal variation of the Lagrangian

$$
\delta \mathscr{L}=-\int d^{4} \theta\left(\bar{D}^{\dot{\alpha}} L^{\alpha} \mathscr{J}_{\alpha \dot{\alpha}}+\bar{D}_{\dot{\beta}} L^{\dot{\beta} \dot{\alpha}} \mathscr{J}_{\dot{\alpha}}\right)+c . c .
$$

directly gives conserved complex currents with conservation equations

$$
\bar{D}^{\dot{\alpha}} \mathscr{J}_{\alpha \dot{\alpha}}=0, \bar{D}_{\dot{\beta}} \mathscr{J}_{\dot{\alpha}}=0 .
$$




\begin{tabular}{lllll}
\hline Shifts & Type A & Type B & Type C & Type D \\
\hline $\mathscr{J}_{\alpha \dot{\alpha}} \rightarrow$ & $\mathscr{J}_{\alpha \dot{\alpha}}+\left[D_{\alpha}, \bar{D}_{\dot{\alpha}}\right] U$ & $\mathscr{J}_{\alpha \dot{\alpha}}+i \partial_{\alpha \dot{\alpha}} U$ & $\mathscr{J}_{\alpha \dot{\alpha}}+\bar{D}_{\dot{\alpha}} D_{\alpha} U$ & $\mathscr{J}_{\alpha \dot{\alpha}}+D_{\alpha} \bar{D}_{\dot{\alpha}} U$ \\
$\mathscr{X}_{\alpha} \rightarrow$ & $\mathscr{X}_{\alpha}-3 \bar{D}^{2} D_{\alpha} U$ & $\mathscr{X}_{\alpha}+\bar{D}^{2} D_{\alpha} U$ & $\mathscr{X}_{\alpha}+2 \bar{D}^{2} D_{\alpha} U$ & $\mathscr{X}_{\alpha}-\bar{D}^{2} D_{\alpha} U$ \\
$\mathscr{Y}_{\alpha} \rightarrow$ & $\mathscr{Y}_{\alpha}-D_{\alpha} \bar{D}^{2} U$ & $\mathscr{Y}_{\alpha}-D_{\alpha} \bar{D}^{2} U$ & $\mathscr{Y}_{\alpha}$ & $\mathscr{Y}_{\alpha}-D_{\alpha} \bar{D}^{2} U$ \\
\hline
\end{tabular}

Table 1: The table presents various shifts which can be used to bring the current to the desired form.

Note that $\mathscr{J}_{\alpha \dot{\alpha}}$ is not necessarily real, therefore

$$
D^{\alpha} \mathscr{J}_{\alpha \dot{\alpha}} \neq 0 .
$$

We may now use improvement terms to bring $\mathscr{J}_{\alpha \dot{\alpha}}, \mathscr{X}_{\alpha}$ and $\mathscr{Y}_{\alpha}$ to the desired form (4.1). These improvement terms can be found in Table 1. After making the current $\mathscr{J}_{\alpha \dot{\alpha}}$ real we find

$$
\begin{aligned}
\mathscr{J}_{\alpha \dot{\alpha}} & =-\frac{1}{2} i \Sigma \partial_{\alpha \dot{\alpha}} \bar{Z}+\frac{1}{2} i \bar{\Sigma} \partial_{\alpha \dot{\alpha}} Z+\frac{1}{2} D^{\beta}\left(i \partial_{\alpha \dot{\alpha}} \Sigma T_{\beta}\right)-\frac{1}{2} \bar{D}^{\dot{\beta}}\left(i \partial_{\alpha \dot{\alpha}} \bar{\Sigma} \bar{T}_{\dot{\beta}}\right), \\
\mathscr{X}_{\alpha} & =\frac{1}{2} \bar{D}^{2} D_{\alpha}(\Sigma \bar{\Sigma}-3 T-Z \bar{\Sigma}-\bar{Z} \Sigma), \\
\mathscr{Y}_{\alpha} & =\frac{1}{2} D_{\alpha} \bar{D}^{2}(\Sigma \bar{\Sigma}-T-Z \bar{\Sigma}-\bar{Z} \Sigma),
\end{aligned}
$$

where

$$
\begin{aligned}
T & =\frac{1}{2 f^{2}}(D \Sigma)^{2}(\bar{D} \bar{\Sigma})^{2}, T_{\beta}=\frac{1}{2 f^{2}} D_{\beta} \Sigma(\bar{D} \bar{\Sigma})^{2}, \bar{T}_{\dot{\beta}}=\frac{1}{2 f^{2}} \bar{D}_{\dot{\beta}} \bar{\Sigma}(D \Sigma)^{2}, \\
Z & =\Sigma+\frac{1}{4 f^{2}} \bar{D}^{\dot{\alpha}}\left(\bar{D}_{\dot{\alpha}} \bar{\Sigma} D^{\alpha} \Sigma D_{\alpha} \Sigma\right) .
\end{aligned}
$$

Moreover, with additional shifts we can still bring the currents into the form of the FZ-multiplet $\left(\mathscr{X}_{\alpha}=0\right)$ or the $\mathscr{R}$-multiplet $\left(\mathscr{Y}_{\alpha}=0\right.$ ) which means that the theory may be consistently coupled to old-minimal or new-minimal supergravity.

Now let us briefly discuss the FZ-multiplet. The supercurrent conservation becomes

$$
\bar{D}^{\dot{\alpha}} \mathscr{J}_{\alpha \dot{\alpha}}=D_{\alpha} X
$$

where $X$ is a chiral superfield. Applying the type A shift with

$$
U=\frac{1}{6}(\Sigma \bar{\Sigma}-3 T-Z \bar{\Sigma}-\bar{Z} \Sigma)
$$

on (4.9), we get a system with a real $\mathscr{J}_{\alpha \dot{\alpha}}$ and

$$
\begin{aligned}
& \mathscr{X}_{\alpha}=0, \\
& \mathscr{Y}_{\alpha}=\frac{2}{3} D_{\alpha} \bar{D}^{2} T .
\end{aligned}
$$


From (4.14) we find that

$$
X=\frac{2}{3} \bar{D}^{2} T
$$

By solving the superspace equation of motion (3.10) have found that $\Sigma=X_{N L}+\bar{\Phi}$, therefore we can insert this in the expression for $X$ (4.15) to find

$$
X=\frac{1}{3 f^{2}} \bar{D}^{2}\left[\left(D X_{N L}\right)^{2}\left(\bar{D} \bar{X}_{N L}\right)^{2}\right]
$$

which gives

$$
X=\frac{1}{3} f X_{N L}
$$

We see that $X$ for the supersymmetry breaking vacuum is proportional to $X_{N L}$, and this will also hold in the IR. Therefore we confirm that $X$ flows to $X_{N L}$ in the IR as was advocated in [21].

To summarize, we have seen that superspace higher derivative terms with complex linear superfields give new possibilities for supersymmetry breaking, with distinct properties, as for example the fact that we get directly a non-linear realization of supersymmetry in the broken vacuum. Future research directions are the coupling of these models to matter superfields and their coupling to supergravity.

\section{Acknowledgments}

It is a pleasure to thank F. Farakos, O. Hulík and R. von Unge for our collaboration on this subject and the organizers of the Corfu Summer Institute 2015 for the very stimulating workshop.

\section{References}

[1] S. J. Gates, M. T. Grisaru, M. Rocek and W. Siegel, "Superspace Or One Thousand and One Lessons in Supersymmetry,” Front. Phys. 58, 1 (1983) [hep-th/0108200].

[2] S. P. Martin, “A Supersymmetry primer,” Adv. Ser. Direct. High Energy Phys. 21, 1 (2010) [Adv. Ser. Direct. High Energy Phys. 18, 1 (1998)] [hep-ph/9709356].

[3] S. J. Gates, Jr. and W. Siegel, "Variant Superfield Representations,” Nucl. Phys. B 187 (1981) 389.

[4] B. B. Deo and S. J. Gates, Jr., "Nonminimal N=1 Supergravity And Broken Global Supersymmetry," Phys. Lett. B 151, 195 (1985). doi:10.1016/0370-2693(85)90833-0

[5] B. B. Deo and S. J. Gates, “Comments On Nonminimal N=1 Scalar Multiplets,” Nucl. Phys. B 254 (1985) 187.

[6] S. J. Gates, Jr., "Why auxiliary fields matter: The Strange case of the 4-D, N=1 supersymmetric QCD effective action,” Phys. Lett. B 365 (1996) 132 [hep-th/9508153].

[7] S. J. Gates, Jr., "Why auxiliary fields matter: The strange case of the 4-D, N=1 supersymmetric QCD effective action. 2.," Nucl. Phys. B 485 (1997) 145 [hep-th/9606109].

[8] M. T. Grisaru, A. Van Proeyen and D. Zanon, "Quantization of the complex linear superfield," Nucl. Phys. B 502 (1997) 345 [hep-th/9703081]. 
[9] S. Penati, A. Refolli, A. Van Proeyen and D. Zanon, "The Nonminimal scalar multiplet: Duality, sigma model, beta function,” Nucl. Phys. B 514 (1998) 460 [hep-th/9710166].

[10] F. Gonzalez-Rey, M. Rocek, S. Wiles, U. Lindstrom and R. von Unge, "Feynman rules in N=2 projective superspace: 1. Massless hypermultiplets," Nucl. Phys. B 516, 426 (1998) doi:10.1016/S0550-3213(98)00073-X [hep-th/9710250].

[11] F. Gonzalez-Rey and R. von Unge, "Feynman rules in N=2 projective superspace. 2. Massive hypermultiplets,” Nucl. Phys. B 516, 449 (1998) doi:10.1016/S0550-3213(98)00074-1 [hep-th/9711135].

[12] S. Cecotti, "Higher Derivative Supergravity Is Equivalent To Standard Supergravity Coupled To Matter. 1.," Phys. Lett. B 190, 86 (1987).

[13] S. Cecotti, S. Ferrara, M. Porrati and S. Sabharwal, "New Minimal Higher Derivative Supergravity Coupled To Matter,” Nucl. Phys. B 306, 160 (1988).

[14] F. Farakos, A. Kehagias and K. Koutrolikos, "Linearized Non-Minimal Higher Curvature Supergravity,” Nucl. Phys. B 894, 569 (2015) [arXiv:1501.07562 [hep-th]].

[15] F. Farakos, S. Ferrara, A. Kehagias and M. Porrati, "Supersymmetry Breaking by Higher Dimension Operators," Nucl. Phys. B 879 (2014) 348 [arXiv:1309.1476 [hep-th]].

[16] F. Farakos and R. von Unge, "Complex Linear Effective Theory and Supersymmetry Breaking Vacua,” Phys. Rev. D 91, no. 4, 045024 (2015) [arXiv:1403.0935 [hep-th]].

[17] F. Farakos, O. Hulík, P. Kočí and R. von Unge, "Non-minimal scalar multiplets, supersymmetry breaking and dualities,” JHEP 1509, 177 (2015) doi:10.1007/JHEP09(2015)177 [arXiv:1507.01885 [hep-th]].

[18] M. Rocek, “Linearizing the Volkov-Akulov Model,” Phys. Rev. Lett. 41, 451 (1978).

[19] U. Lindstrom and M. Rocek, "Constrained Local Superfields," Phys. Rev. D 19, 2300 (1979).

[20] R. Casalbuoni, S. De Curtis, D. Dominici, F. Feruglio and R. Gatto, "Nonlinear Realization of Supersymmetry Algebra From Supersymmetric Constraint,” Phys. Lett. B 220, 569 (1989).

[21] Z. Komargodski and N. Seiberg, "From Linear SUSY to Constrained Superfields,” JHEP 0909, 066 (2009) [arXiv:0907.2441 [hep-th]].

[22] S. Cecotti, S. Ferrara and L. Girardello, "Structure of the Scalar Potential in General $N=1$ Higher Derivative Supergravity in Four-dimensions,” Phys. Lett. B 187, 321 (1987). doi:10.1016/0370-2693(87)91103-8

[23] I. L. Buchbinder, S. Kuzenko and Z. Yarevskaya, "Supersymmetric effective potential: Superfield approach," Nucl. Phys. B 411, 665 (1994).

[24] I. L. Buchbinder, S. M. Kuzenko and A. Y. Petrov, "Superfield chiral effective potential," Phys. Lett. B 321, 372 (1994).

[25] M. Rocek and A. A. Tseytlin, "Partial breaking of global D = 4 supersymmetry, constrained superfields, and three-brane actions,” Phys. Rev. D 59, 106001 (1999) [hep-th/9811232].

[26] F. Gonzalez-Rey, I. Y. Park and M. Rocek, "On dual 3-brane actions with partially broken N=2 supersymmetry,” Nucl. Phys. B 544, 243 (1999) [hep-th/9811130].

[27] J. Khoury, J. L. Lehners and B. Ovrut, "Supersymmetric P(X, $\phi)$ and the Ghost Condensate," Phys. Rev. D 83, 125031 (2011) [arXiv:1012.3748 [hep-th]]. 
[28] M. Koehn, J. L. Lehners and B. A. Ovrut, "Higher-Derivative Chiral Superfield Actions Coupled to N=1 Supergravity,” Phys. Rev. D 86, 085019 (2012) [arXiv:1207.3798 [hep-th]].

[29] F. Farakos and A. Kehagias, "Emerging Potentials in Higher-Derivative Gauged Chiral Models Coupled to N=1 Supergravity," JHEP 1211, 077 (2012) [arXiv:1207.4767 [hep-th]].

[30] C. Adam, J. M. Queiruga, J. Sanchez-Guillen and A. Wereszczynski, "Extended Supersymmetry and BPS solutions in baby Skyrme models,” JHEP 1305, 108 (2013) [arXiv:1304.0774 [hep-th]].

[31] M. Nitta and S. Sasaki, "BPS States in Supersymmetric Chiral Models with Higher Derivative Terms,” Phys. Rev. D 90, no. 10, 105001 (2014) [arXiv:1406.7647 [hep-th]].

[32] M. Nitta and S. Sasaki, "Higher Derivative Corrections to Manifestly Supersymmetric Nonlinear Realizations,” Phys. Rev. D 90, no. 10, 105002 (2014) [arXiv:1408.4210 [hep-th]].

[33] E. Dudas and D. M. Ghilencea, "Effective operators in SUSY, superfield constraints and searches for a UV completion,” JHEP 1506, 124 (2015) doi:10.1007/JHEP06(2015)124 [arXiv:1503.08319 [hep-th]].

[34] M. Nitta and S. Sasaki, "Classifying BPS States in Supersymmetric Gauge Theories Coupled with Higher Derivative Chiral Models," arXiv:1504.08123 [hep-th].

[35] D. Ciupke, J. Louis and A. Westphal, "Higher-Derivative Supergravity and Moduli Stabilization," arXiv:1505.03092 [hep-th].

[36] J. M. Queiruga, "Skyrme-like models and supersymmetry in 3+1 dimensions," Phys. Rev. D 92 , no. 10, 105012 (2015) doi:10.1103/PhysRevD.92.105012 [arXiv:1508.06692 [hep-th]].

[37] S. Bielleman, L. E. Ibanez, F. G. Pedro, I. Valenzuela and C. Wieck, "The DBI Action, Higher-derivative Supergravity, and Flattening Inflaton Potentials,” arXiv:1602.00699 [hep-th].

[38] S. M. Kuzenko and S. J. Tyler, “Complex linear superfield as a model for Goldstino,” JHEP 1104 (2011) 057 [arXiv:1102.3042 [hep-th]].

[39] S. Ferrara and B. Zumino, “Transformation Properties of the Supercurrent,” Nucl. Phys. B 87, 207 (1975).

[40] M. Magro, I. Sachs and S. Wolf, "Superfield Noether procedure,” Annals Phys. 298, 123 (2002) [hep-th/0110131].

[41] Z. Komargodski and N. Seiberg, "Comments on Supercurrent Multiplets, Supersymmetric Field Theories and Supergravity,” JHEP 1007, 017 (2010) [arXiv:1002.2228 [hep-th]].

[42] S. M. Kuzenko, "Variant supercurrent multiplets," JHEP 1004, 022 (2010) [arXiv:1002.4932 [hep-th]].

[43] S. M. Kuzenko, "Variant supercurrents and Noether procedure," Eur. Phys. J. C 71, 1513 (2011) [arXiv:1008.1877 [hep-th]]. 